\title{
A Patient with Postpartum Hypopituitarism (Sheehan's Syndrome) Developed Postpartum Autoimmune Thyroiditis (Transient Thyrotoxicosis and Hypothyroidism): A Case Report and Review of the Literature
}

\author{
Nobuyuki Takasu and Yoshirou Nakayama \\ Department of Endocrinology and Metabolism, Aizawa Hospital, 2-5-1 Honjo, Mtasumoto 390-8521, Japan \\ Correspondence should be addressed to Nobuyuki Takasu, nobuyukitakasu@gmail.com
}

Received 25 August 2010; Revised 15 January 2011; Accepted 14 February 2011

Academic Editor: Gary L. Francis

Copyright (C) 2011 N. Takasu and Y. Nakayama. This is an open access article distributed under the Creative Commons Attribution License, which permits unrestricted use, distribution, and reproduction in any medium, provided the original work is properly cited.

\begin{abstract}
A 36-year-old woman with postpartum hypopituitarism (Sheehan's syndrome: SS) developed postpartum autoimmune thyroiditis (PPAT). She delivered a baby by Caesarean section $(620 \mathrm{~mL}$ blood loss). At 1 month post partum, she developed thyrotoxicosis due to painless thyroiditis (autoimmune destructive thyroiditis). She was positive for antithyroid antibodies. Postpartum and hypoadrenalism-induced exacerbation of autoimmune thyroiditis caused the thyrotoxicosis due to autoimmune destructive thyroiditis. ACTH was undetectable. She had ACTH deficiency and secondary hypoadrenalism. Hydrocortisone was started. At 6 months post partum, she was referred to us with hypothyroidism. Thyroxine was administered. She had thyrotoxicosis at 12 months post partum and then hypothyroidism. She was diagnosed with PPAT. She had hypopituitarism, ACTH deficiency (secondary hypoadrenalism), low prolactin with agalactia, and low LH with failure to resume regular menses. She had empty sella on MRI. She was diagnosed with SS. Three cases with SS have been reported to develop PPAT. Postpartum immunological rebounds and hypoadrenalism-induced immunological alterations (or a combination of the two) might have been responsible for the PPAT.
\end{abstract}

\section{Introduction}

Sheehan's syndrome (SS), first described by Sheehan in 1937 [1], is postpartum hypopituitarism caused by intrapartum or postpartum hemorrhage. SS may cause partial or complete hypopituitarism. It may also cause secondary hypoadrenalism. Pregnancy and delivery have a profound effect on autoimmune thyroid diseases during gestation and the postpartum period [2]. Postpartum transient thyrotoxicosis and hypothyroidism have been reported [3, 4]. They are postpartum exacerbation or development of autoimmune thyroiditis and have been called postpartum autoimmune thyroiditis (PPAT), postpartum thyroiditis, or postpartum painless thyroiditis [3-8]. PPAT, postpartum thyroiditis, or postpartum painless thyroiditis is a member of autoimmune thyroiditis (Hashimoto's thyroiditis)
[9]. The exacerbation and development of autoimmune thyroiditis have also been reported after adrenalectomy in patients with Cushing's syndrome $[10,11]$. The decrease in cortisol after adrenalectomy exacerbates autoimmune thyroiditis. Exacerbation of autoimmune thyroiditis has been also reported after cessation of steroid therapy in a patient with autoimmune thyroiditis and rheumatoid arthritis [12]. Three cases with SS have been reported to develop PPAT [5, $13,14]$. A case with transient thyrotoxicosis due to painless thyroiditis (autoimmune destructive thyroiditis) following pituitary apoplexy was also reported [15]. Pituitary apoplexy and SS may cause secondary hypoadrenalism or a serum cortisol decrease. This decrease in cortisol may exacerbate autoimmune thyroid diseases. Steroid hormones decrease after delivery. Postpartum steroid hormone decrease may exacerbate autoimmune thyroid diseases. 
We encountered a patient with postpartum hypopituitarism (Sheehan's syndrome: SS), who developed postpartum autoimmune thyroiditis (PPAT) (transient thyrotoxicosis and hypothyroidism). Postpartum immunological rebounds and hypoadrenalism-induced immunological alterations (or a combination of the two) might have been responsible for the development of PPAT in this patient.

\section{Materials and Methods}

2.1. Hormone Assays. Serum TSH, free T3, free T4, total T3, total T4, thyroglobulin, antithyroid peroxidase antibody (TPOAb), antithyroglobulin antibody (TGAb), progesterone, estradiol, serum prolactin, and plasma ACTH were determined by electrochemiluminescence immunoassays (ECLIA) (Roche Diagnostics, Tokyo, Japan). The intraassay coefficient of variation (CV) was $2.1 \%, 3.5 \%, 5.2 \%, 4.3 \%$, $3.2 \%, 5.1 \%, 5.1 \%, 6.5 \%, 4.5 \%, 3.3 \%, 3.1 \%$, and $3.6 \%$, respectively, and interassay CV was 3.5\%, 8.4\%, 9.4\%, 9.4\%, $8.2 \%, 7.8 \%, 9.4 \%, 10.6 \%, 9.2 \%, 6.4 \%, 6.5 \%$, and $7.2 \%$, respectively. Serum TSH receptor antibody (TRAb) (TRAb (human)) was determined by a radioreceptor assay (RRA) (Yamasa Co., Tokyo, Japan). The intraassay CV was 7.6\%, and interassay CV was $12.4 \%$. Serum cortisol, GH, IGF-1, and urinary cortisol were measured by radioimmunoassay (RIA) (TFB, Inc., Tokyo, Japan). The intraassay CV was $5.8 \%, 3.3 \%, 3.0 \%$, and $6.8 \%$, respectively, and interassay CV was $8.9 \%, 6.5 \%, 6.2 \%$, and $10.2 \%$, respectively. LH and FSH were measured by chemiluminescence immunoassay (CLIA) (Abbott Lab., Tokyo, Japan). The intraassay CV was 3.5\% and $3.3 \%$, respectively, and interassay $\mathrm{CV}$ was $6.5 \%$ and $7.2 \%$, respectively. Plasma $\mathrm{ADH}$ was measured by RIA (Mitsubishi Chemical Medicine Corp., Tokyo, Japan). The intraassay CV was $6.1 \%$, and interassay CV was $9.5 \%$. Hormone assays were performed at the SRL Institute (Tokyo, Japan). Normal reference ranges for hormone concentrations are described in the tables, legends for figures, or elsewhere as cited.

2.2. Endocrine and Other Studies. A thyrotropin-releasing hormone (TRH) test, using $500 \mu \mathrm{g}$ TRH, was performed to estimate TSH and prolactin secretion. The test was done in the morning after an overnight fast. Samples for TSH and prolactin were drawn at $0,30,60,90$, and 120 minutes after intravenous TRH administration. Peak TSH and prolactin levels occur at 30 minutes in normal subjects.

A corticotrophin-releasing hormone $(\mathrm{CRH})$ test, using $100 \mu \mathrm{g}$ CRH (human CRH: Corticorelin), was performed to estimate ACTH secretion. The test was done in the morning after an overnight fast. The patient was on bed rest for at least 40 minutes before the first blood sample was drawn. Plasma ACTH and serum cortisol levels were measured at 0, 30, 60, 90, and 120 minutes after the intravenous $\mathrm{CRH}$ injection. Peak ACTH levels occur at 30-60 minutes in healthy subjects, while a lack of ACTH secretion is seen in patients with pituitary ACTH insufficiency.

A gonadotropin-releasing hormone $(\mathrm{GnRH})$ test, using $100 \mu \mathrm{g}$ GnRH (LH-RH), was performed to estimate LH and FSH secretion. The test was done in the morning after an overnight fast. Samples for LH and FSH were drawn at $0,30,60,90$, and 120 minutes after the intravenous $\mathrm{GnRH}$ injection. Gonadotropin deficiency was diagnosed by subnormal LH and FSH responses to GnRH. TRH, CRH, and GnRH tests were performed separately.

An insulin tolerance test (ITT) was used to test growth hormone $(\mathrm{GH})$ secretion. The test was performed in the morning after an overnight fast. Five-unit insulin was given intravenously, and glucose and $\mathrm{GH}$ concentrations were measured at $-30,0,30,60,90$, and 120 minutes. GH deficiency was defined by a peak GH response of less than $3 \mu \mathrm{g} / \mathrm{L}$ with low concentrations of IGF-I [16].

Written informed consent was obtained from the patient prior to publication of this paper.

\section{Case Report}

A 36-year-old Japanese woman was referred to us at 6 months post partum with easy fatigability and agalactia (Figure 1 and Table 1, 6 months). She had delivered a full-term baby by Caesarean section (Figure 1, Delivery). Her blood-loss was estimated to be $620 \mathrm{~mL}$. During the delivery, she did not have hypotension and remained normotensive. She was discharged without any apparent complications. However, she began to complain of easy fatigability, lassitude, agalactia, and loss of appetite after the delivery.

She visited a doctor at 1 month post partum (Figure 1 and Table 1, 1 month). A physical examination at that time revealed a supine blood pressure of 90/48 $\mathrm{mmHg}$. Her pulse rate was $122 / \mathrm{min}$ and temperature was $37.2^{\circ} \mathrm{C}$. An examination showed moist skin and finger tremors with clear lungs and a soft abdomen. Exophthalmoses were not observed. She had thyrotoxicosis clinically. A thyroid function study demonstrated that she had thyrotoxicosis; serum free T3 and free T4 levels were elevated, and serum TSH levels were undetectable (Figure 1 and Table 1, 1-2 months). She was negative for TRAb. However, she was positive for TPOAb and TGAb. At 1 month ante partum, her TPOAb was $3.4 \mathrm{kIU} / \mathrm{L}$ and her TGAb was $52.0 \mathrm{kIU} / \mathrm{L}$ (Table 1 ). At 2 months post partum, her TPOAb had increased to $42.2 \mathrm{kIU} / \mathrm{L}$ and her TGAb had increased to $138.4 \mathrm{kIU} / \mathrm{L}$. Her serum thyroglobulin was $72 \mu \mathrm{g} / \mathrm{L}$ (normal $<32 \mu \mathrm{g} / \mathrm{L}$ ), radioactive iodine uptake was $0.5 \% / 24 \mathrm{hr}$ (normal 10-40\%), and thyroid scanning with radioiodine showed no detectable uptake. She had thyrotoxicosis due to autoimmune destructive thyroiditis. Her plasma ACTH was less than $0.4 \mathrm{pmol} / \mathrm{L}$, and her serum cortsol was less than $5.5 \mathrm{nmol} / \mathrm{L}$. She therefore had ACTH deficiency and secondary hypoadrenalism; $20 \mathrm{mg}$ hydrocortisone (HC) was started (Figure 1, 1 month post partum). The thyrotoxicosis, due to autoimmune destructive thyroiditis, subsided spontaneously. She became euthyroid. At 4 months post partum, she had hypothyroidism with a serum TSH of $6.6 \mathrm{mIU} / \mathrm{L}$ (Figure 1 and Table 1, 4 months).

At 6 months post partum, she was referred to us with easy fatigability and agalactia (Figure 1 and Table 1, 6 months). On admission, she was well oriented and fully conscious. Her height was $163 \mathrm{~cm}$, and her weight was $52.7 \mathrm{~kg}$. She was afebrile with a temperature of $36.5^{\circ} \mathrm{C}$. Her blood pressure 


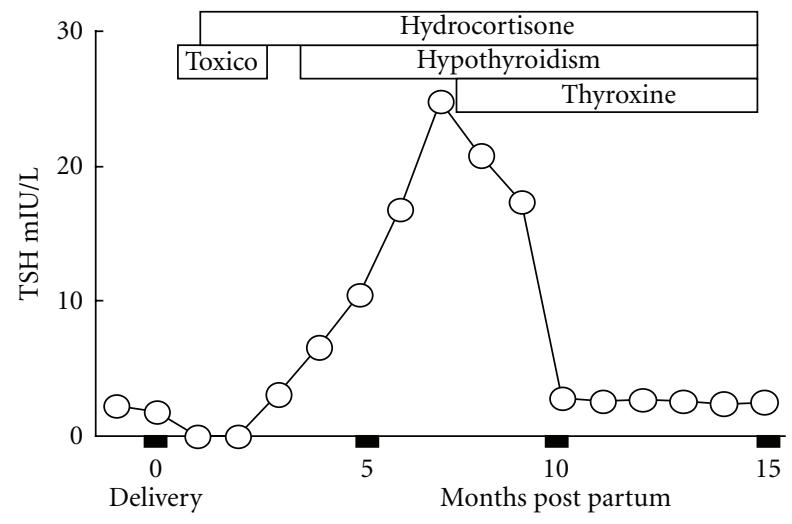

TSH mIU/L

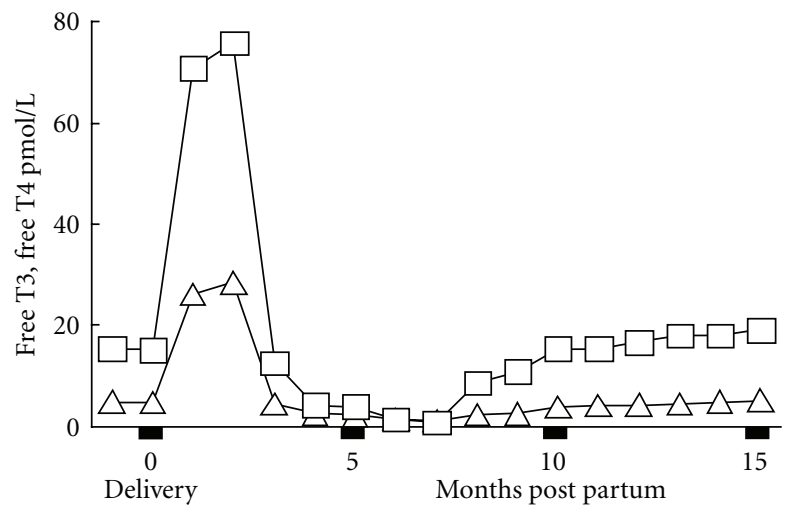

$\triangle$ Free T3 pmol/L

Free T4 pmol/L

FIGURE 1: The clinical course of a patient with postpartum hypopituitarism (Sheehan's syndrome: SS), who developed postpartum autoimmune thyroiditis (PPAT) (transient thyrotoxicosis and hypothyroidism). A 36-year-old woman delivered a full-term baby by Caesarean section (Delivery). At 1 month post partum, she visited a doctor with thyrotoxicosis (Toxico). She was negative for TRAb. However, she was positive for TPOAb and TGAb. TPOAb- and TGAb-titers increased after delivery. Her serum thyroglobulin was $72 \mu \mathrm{g} / \mathrm{L}$ (normal $<32 \mu \mathrm{g} / \mathrm{L}$ ). Radioactive iodine uptake was $0.5 \% / 24 \mathrm{hr}$ (normal 10-40\%). She had thyrotoxicosis (Toxico) due to painless thyroiditis (autoimmune destructive thyroiditis). Her ACTH was less than $0.4 \mathrm{pmol} / \mathrm{L}$, and her cortisol was less than $5.5 \mathrm{nmol} / \mathrm{L}$. She had ACTH deficiency and secondary hypoadrenalism; $20 \mathrm{mg}$ hydrocortisone (HC) was started. The thyrotoxicosis subsided spontaneously. At 4 months post partum, she developed hypothyroidism (hypothyroidism) with TSH $6.6 \mathrm{mIU} / \mathrm{L}$. At 6 months post partum, she was referred to us with easy fatigability and agalactia. She had hypothyroidism with TSH $16.8 \mathrm{mIU} / \mathrm{L}$. She had thyrotoxicosis (Toxico) at 1-2 months post partum and then hypothyroidism (hypothyroidism) (PPAT). At 7 months, thyroxine (T4) was started. She had hypopituitarism and empty sella on MRI (SS). She is now taking $75 \mu \mathrm{g} \mathrm{T} 4$ and $20 \mathrm{mg} \mathrm{HC}$ daily. Normal reference ranges: TSH $0.4-4.20 \mathrm{mIU} / \mathrm{L}$, free T3 (free triiodothyronine) $3.5-6.6 \mathrm{nmol} / \mathrm{L}$, and free T4 (free thyroxine) $11.6-21.9 \mathrm{pmol} / \mathrm{L}$.

TABLE 1: Results of thyroid and adrenal function tests and TPOAb and TGAb at 1 month before delivery ( $-1 \mathrm{~m})(1 \mathrm{month}$ ante partum $)$ and 1-10 months after delivery (1-10 $\mathrm{m})(1-10$ months post partum).

\begin{tabular}{|c|c|c|c|c|c|c|c|c|c|}
\hline Months $(\mathrm{m})^{*}$ & $-1 \mathrm{~m}$ & $1 \mathrm{~m}$ & $2 \mathrm{~m}$ & $4 \mathrm{~m}$ & $6 \mathrm{~m}$ & $7 \mathrm{~m}$ & $8 \mathrm{~m}$ & $9 \mathrm{~m}$ & $10 \mathrm{~m}$ \\
\hline Free T3 pmol/L & 4.9 & 26.2 & 28.5 & 2.5 & 1.5 & 1.2 & 2.3 & 2.5 & 3.9 \\
\hline Free T4 pmol/L & 15.4 & 70.8 & 75.9 & 4.3 & 1.4 & 0.9 & 8.7 & 10.9 & 15.4 \\
\hline TSH mIU/L & 2.2 & $<0.005$ & $<0.005$ & 6.6 & 16.8 & 24.8 & 20.8 & 17.4 & 2.8 \\
\hline ACTH pmol/L & 11.5 & $<0.4$ & $<0.4^{* *}$ & & $<0.4^{* *}$ & & & & \\
\hline Cortisol nmol/L & 690 & $<5.5$ & $<5.5^{* *}$ & & $<5.5^{* *}$ & & & & \\
\hline TPOAb kIU/L & 3.4 & & 42.2 & & 34.1 & & & & 26.8 \\
\hline TGAb kIU/L & 52.0 & & 138.4 & & 126.7 & & & & 102.3 \\
\hline TRAb IU/L & 0.3 & & 0.4 & & 0.5 & & & & 0.4 \\
\hline
\end{tabular}

Months $(\mathrm{m})^{*}:-1 \mathrm{~m}$ : 1 month before delivery $\left(1\right.$ month ante partum) and 1-10 m: 1-10 months after delivery (1-10 months post partum). ${ }^{*}$ Oral hydrocortisone (HC) had been discontinued for 1 week before the study. Free T3: free triiodothyronine, free T4: free thyroxine, TPOAb: antithyroid peroxidase antibody, TGAb: antithyroglobulin antibody, and TRAb: TSH receptor antibody. Normal reference ranges: free T3 3.5-6.6 nmol/L, free T4 11.6-21.9 pmol/L, TSH 0.4-4.20 mIU/L, ACTH 1.70-12.27 pmol/L, cortisol 110-505 nmol/L, TPOAb $<0.3 \mathrm{kIU} / \mathrm{L}, \mathrm{TGAb}<0.3 \mathrm{kIU} / \mathrm{L}$, and TRAb (TRAb (human)) < 1.0 IU/L. A patient with postpartum hypopituitarism (SS) developed postpartum autoimmune thyroiditis (PPAT). At 1 month post partum, she had thyrotoxicosis. She was negative for TRAb. She was positive for TPOAb and TGAb. TPOAb- and TGAb-titers increased after delivery. Serum thyroglobulin was $72 \mu \mathrm{g} / \mathrm{L}$ (normal $<32 \mu \mathrm{g} / \mathrm{L}$ ). She had painless thyroiditis (autoimmune destructive thyroiditis). She had thyrotoxicosis due to destructive thyroiditis. ACTH was less than $0.4 \mathrm{pmol} / \mathrm{L}$ and cortisol was less than $5.5 \mathrm{nmol} / \mathrm{L}$. She had ACTH deficiency and secondary hypoadrenalism; HC was started. At 4 months, she had hypothyroidism with TSH $6.6 \mathrm{mIU} / \mathrm{L}$. At 6 months, TSH was $16.8 \mathrm{mIU} / \mathrm{L}$. At 7 months, thyroxine (T4) was started. She had thyrotoxicosis at 1-2 months post partum and then hypothyroidism (PPAT). She had hypopituitarism (SS). She is now taking T4 and HC.

was 100/64 mmHg. Her pulse rate was $72 / \mathrm{min}$. Her skin was not moist. She had a goiter and delayed deep tendon reflexes. She had sparse axillary and pubic hairs. She had atrophy of the breasts and agalactia. She had also failed to resume regular menses after delivery. Systemic examination did not indicate any other abnormalities. She had hypothyroidism with a serum TSH of $16.8 \mathrm{mIU} / \mathrm{L}$ (Figure 1 and Table 1, 6 months). Figure 1 shows her clinical course. Since she had thyrotoxicosis at 1-2 months post partum and then developed hypothyroidism, she was diagnosed to have postpartum 


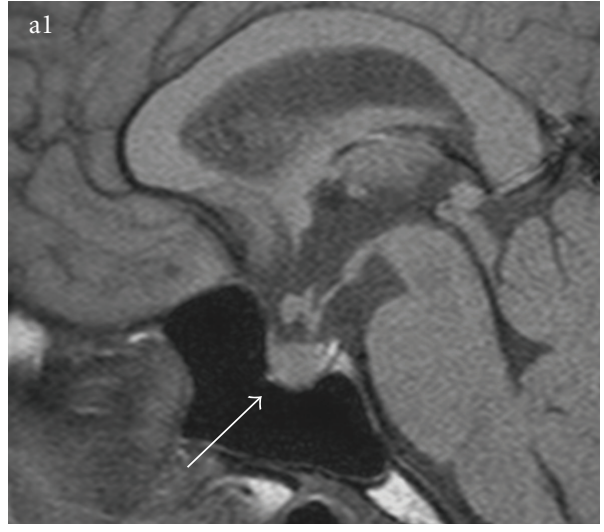

T1WI sagittal

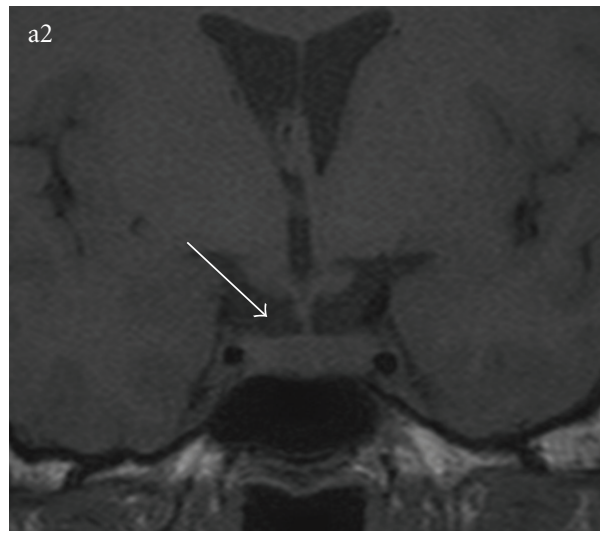

T1WI coronal

(a) 2 months post partum

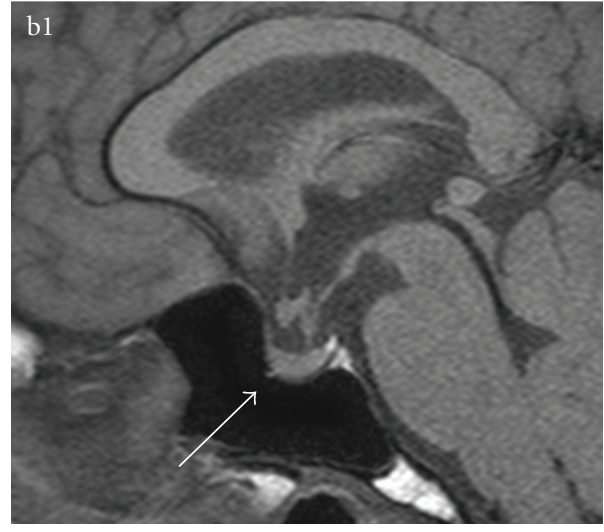

T1WI sagittal

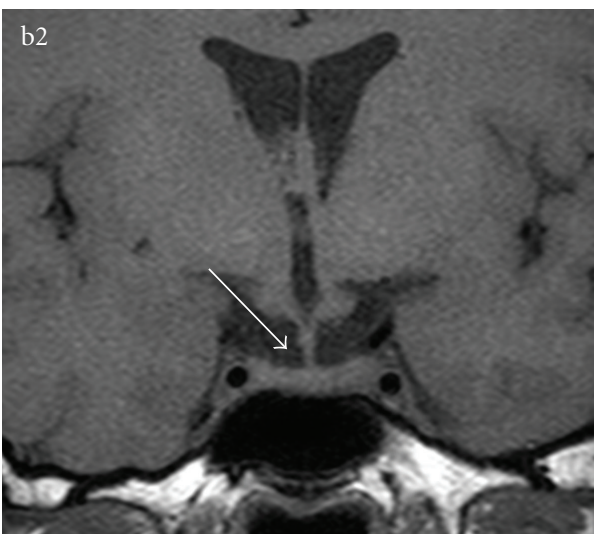

T1WI coronal

(b) 6 months post partum

FIGURE 2: Sequential magnetic resonance imaging (MRI) (T1 weighted image: T1WI) demonstrated changes of the pituitary gland. At 2 months post partum, MRI revealed a normal pituitary gland ((a1) T1WI sagittal, (a2) T1WI coronal). At 6 months post partum, MRI revealed atrophy of the pituitary gland and empty sella ((b1) T1WI sagittal, (b2) T1WI coronal). At 6 months post partum, she had empty sella on MRI. The arrows indicate the pituitary gland.

autoimmune thyroiditis (PPAT). At 7 months post partum, thyroxine (T4) was started. She is now taking $75 \mu \mathrm{g}$ T4 and 20 mg HC daily.

The patient had adrenal insufficiency. The laboratory evaluation at 6 months post partum had revealed that she had low plasma ACTH, low serum cortisol (Table 1, 6 months, Table 2), and low urinary cortisol (Table 3(a)). Table 3(b) demonstrates that she had no ACTH response to CRH. She therefore had ACTH deficiency and secondary hypoadrenalism.

She had agalactia. She had low serum prolactin (Tables 2 and $3(\mathrm{~b})$ ) and a low prolactin response to TRH (Table 3(b)). She had failed to resume regular menses after delivery. She had low LH (Tables 2 and 3(b)) and delayed LH and FSH responses to GnRH (Table 3(b)).

At 6 months, she had hypothyroidism with a serum TSH of $16.8 \mathrm{mIU} / \mathrm{L}$ (Figure 1 and Table 1, 6 months, Table 2). The elevated TSH levels indicated that she had primary hypothyroidism. She was positive for TPOAb and TGAb. She therefore had primary hypothyroidism due to autoimmune thyroiditis. Her TSH levels were high, her TSH response to
TRH was delayed, and the magnitude of the TSH response to TRH was low (Table 3(b)), indicating that the pituitary TSH response to TRH might be impaired. It is possible that she had pituitary TSH secretion failure due to SS, in addition to primary hypothyroidism due to autoimmune thyroiditis [17].

The GH response to ITT was blunted (Table 3(c)) and the IGF-1 level was in the lower normal range (Table 2). The patient therefore had partial GH deficiency. She did not have diabetes insipidus (Table 3(d)).

The patient was determined to have hypopituitarism, ACTH deficiency (secondary hypoadrenalism), low prolactin with agalactia, and low LH with failure to resume regular menses. Sequential magnetic resonance imaging (MRI) demonstrated changes in the pituitary gland. At 2 months post partum, MRI had revealed a normal pituitary gland (Figure 2(a)), while, at 6 months, MRI revealed atrophy of the pituitary gland and empty sella (Figure 2(b)).

In summary, a patient with postpartum hypopituitarism (Sheehan's syndrome: SS) developed postpartum autoimmune thyroiditis (PPAT). She had hypopituitarism (ACTH 
deficiency, low prolactin, and low LH) and empty sella on MRI (SS). She developed transient thyrotoxicosis at 1-2 months post partum and then subsequent hypothyroidism (PPAT).

\section{Discussion}

A patient with postpartum hypopituitarism (Sheehan's syndrome: SS) developed postpartum autoimmune thyroiditis (PPAT). After delivery, she had agalactia and failure to resume regular menses and was found to have hypopituitarism and empty sella on MRI (SS). She had transient thyrotoxicosis at 1-2 months post partum and then developed hypothyroidism (PPAT). Postpartum immunological rebounds and hypoadrenalism-induced immunological alterations (or a combination of the two) may have been responsible for the development of PPAT.

SS may cause secondary hypoadrenalism. SS is characterized by a wide spectrum of clinical features. In the past, hypothyroidism in the postpartum period was considered in the context of hypopituitarism due to SS. However, studies over the past several decades have altered this concept. Exacerbation or development of autoimmune thyroid diseases has been reported to occur among women after uneventful delivery and has been called postpartum autoimmune thyroiditis (PPAT), postpartum thyroiditis, or postpartum painless thyroiditis (postpartum autoimmune destructive thyroiditis) [3-8]. PPAT, postpartum thyroiditis, or postpartum painless thyroiditis is a member of a group of autoimmune thyroiditis (Hashimoto's thyroiditis) [9].

PPAT may involve thyrotoxicosis or hypothyroidism. The first phase is typically thyrotoxicosis due to autoimmune destructive thyroiditis (painless thyroiditis). Then, the thyroid function returns to normal. Some patients may subsequently develop hypothyroidism. Three cases with postpartum hypopituitarism (SS) have been reported to develop postpartum autoimmune thyroiditis (PPAT) $[5,13$, 14].

The criteria for the diagnosis of SS are as follows: (1) typical obstetric history of intrapartum or postpartum bleeding, (2) hypotension or shock, (3) agalactia, (4) failure to resume regular menses after delivery, (5) hypopituitarism, and (6) empty sella on CT or MRI [18]. Our patient had (3), (4), (5), and (6). Kaplun et al. [19] reported that sequential MRI demonstrates evidence of ischemic infarct in the pituitary gland with enlargement, followed by gradual shrinkage to pituitary atrophy. In our case, MRI revealed atrophy of the pituitary gland and empty sella at 6 months post partum.

SS is described as postpartum hypopituitarism due to pituitary necrosis caused by hypotension or shock secondary to massive bleeding during or just after delivery. The exact pathogenesis and natural history are not understood [18]. The role of autoimmunity, including pituitary autoimmunity, in the development of SS has been also suggested, since pituitary autoantibody positivity is significantly higher in SS patients than controls $[20,21]$. In SS, pituitary CT or MRI reveals an empty sella, similar to our patient.
TABLe 2: Fasting blood hormone levels at 9:00 (6 months post partum)*.

\begin{tabular}{lc}
\hline & $\begin{array}{c}\text { Fasting hormone levels at 9:00(normal } \\
\text { references) }\end{array}$ \\
\hline ACTH pmol/L & $<0.4(1.7-12.3)$ \\
Cortisol nmol/L & $<5.5(110.4-504.9)$ \\
TSH mIU/L & $16.8(0.4-4.20)$ \\
$\mathrm{fT} 3 \mathrm{pmol} / \mathrm{L}[\mathrm{tT} 3$ & $1.54(3.54-6.62)[0.97(1.23-2.46)]$ \\
$\mathrm{nmol} / \mathrm{L}]$ & \\
$\mathrm{fT} 4 \mathrm{pmol} / \mathrm{L}[\mathrm{tT} 4$ & $1.42(11.58-21.88)[12.9(78.5-159.6)]$ \\
$\mathrm{nmol} / \mathrm{L}]$ & $104(266-1328)$ \\
Prolactin pmol/L & $1.0(0.28-1.64)$ \\
GH $\mu \mathrm{g} / \mathrm{L}$ & $112(73-311)$ \\
IGF-1 $\mu \mathrm{g} / \mathrm{L}$ & $0.7(1.13-14.22)$ \\
LH IU/L & $2.9(1.47-8.49)$ \\
FSH IU/L & $98.5(4.07-98.8)$ \\
Progesterone & $607.6(165.2-1101.3)$ \\
nmol/L &
\end{tabular}
the study. fT3: free triiodothyronine, tT3: total triiodothyronine, fT4: free thyroxine, and tT4: total thyroxine.

Komatsu et al. [22] reported that serum antipituitary antibodies were positive in $70 \%$ of patients with empty sella and suggested that antipituitary antibodies might be related to the development of pituitary atrophy and the consequent empty sella. Our patient had positive tests for TPOAb and TGAb, indicating that she had had autoimmune thyroiditis. Both obstetric hemorrhage and autoimmune processes may affect postpartum hypopituitarism. Immune process might also be involved in the development of SS [20,21].

Postpartum autoimmune thyroiditis (PPAT) is believed to result from the modifications of the immune system during pregnancy and delivery. PPAT may involve thyrotoxicosis and/or hypothyroidism. The first phase is typically thyrotoxicosis due to autoimmune destructive thyroiditis. Patients with PPAT have positive TPOAb and/or TGAb. They may have recurrences after each pregnancy and may eventually develop hypothyroidism. Any woman who develops PPAT should be carefully followed up, as she has an increased risk of developing hypothyroidism.

Three cases with postpartum hypopituitarism (SS) have been reported to develop postpartum autoimmune thyroiditis (PPAT) $[5,13,14]$ (Table 4). We herein reported another case of SS, who developed PPAT. All four cases had positive TPOAb and/or TGAb. They initially presented with transient thyrotoxicosis due to autoimmune destructive thyroiditis. Three of the four patients were Japanese. The postpartum changes of autoimmune system may be responsible for the development of PPAT. SS also causes secondary hypoadrenalism, which causes a decrease in cortisol. The cortisol decrease can then induce immunological alterations. Postpartum immunological rebounds and hypoadrenalisminduced immunological alterations (or a combination of the two) might therefore be the underlying pathogenesis 
TABLE 3: Endocrine studies at 6 months post partum.

(a) Urinary cortisol (studied one week after discontinuation of hydrocortisone)

\begin{tabular}{|c|c|c|c|c|c|c|}
\hline & & & & & \multicolumn{2}{|c|}{ (Normal references) } \\
\hline \multicolumn{5}{|c|}{ Urinary cortisol nmol/day } & \multicolumn{2}{|c|}{$<18(30-230)$} \\
\hline & $0 \mathrm{~min}$ & $30 \mathrm{~min}$ & $60 \mathrm{~min}$ & $90 \mathrm{~min}$ & $120 \mathrm{~min}$ & Response \\
\hline \multicolumn{7}{|c|}{ CRH test (studied one week after discontinuation of hydrocortisone) } \\
\hline ACTH pmol/L & $<0.4$ & $<0.4$ & $<0.4$ & $<0.4$ & $<0.4$ & No \\
\hline Cortisol nmol/L & $<5.5$ & $<5.5$ & $<5.5$ & $<5.5$ & $<5.5$ & No \\
\hline \multicolumn{7}{|l|}{ TRH test } \\
\hline TSH mIU/L & 17.4 & 38.1 & 52.9 & 57.1 & 57.7 & Delayed \\
\hline Prolactin $\mathrm{pmol} / \mathrm{L}$ & 43.5 & 178.3 & 160.9 & 165.2 & 139.1 & Low \\
\hline \multicolumn{7}{|l|}{ GnRH test } \\
\hline LH IU/L & 0.5 & 6.6 & 11.1 & 13.1 & 13.7 & Delayed \\
\hline FSH IU/L & 2.2 & 2.6 & 3.3 & 3.9 & 4.3 & Delayed \\
\hline
\end{tabular}

Normal references for basal ACTH, cortisol, TSH, prolactin, LH, and FSH appear in Table 2.

(c) Insulin tolerance test (ITT) for GH releases

\begin{tabular}{lcccccc}
\hline & $-30 \mathrm{~min}$ & $0 \mathrm{~min}$ & $30 \mathrm{~min}$ & $60 \mathrm{~min}$ & $90 \mathrm{~min}$ & 3.8 \\
$\mathrm{GH} \mu \mathrm{g} / \mathrm{L}$ & 1.0 & 1.1 & 0.8 & 4.1 & $\mathrm{~min}$ \\
$\mathrm{BG} \mathrm{nmol} / \mathrm{L}$ & 4.6 & 4.3 & 2.2 & 3.1 & 3.7 & 2.5 \\
\hline
\end{tabular}

BG: blood glucose. Normal references for basal GH appear in Table 2.

(d) $\mathrm{ADH}$ (antidiuretic hormone), plasma osmolality, and urine osmolality at 9:00

\begin{tabular}{lc}
\hline & (Normal references) \\
\hline Plasma ADH pmol/L & $0.92(0.28-3.23)$ \\
Plasma osmolality mmol/kg & $287(285-293)$ \\
Urine osmolality mmol/kg & $767(300-900)$ \\
\hline
\end{tabular}

TABLE 4: Postpartum hypopituitarism (Sheehan's syndrome: SS) and postpartum autoimmune thyroiditis (PPAT): a review of the literature.

\begin{tabular}{lcccccc}
\hline Case & Age, yr & Nationality & ATA* & Thyroid state** & Publication year & Reference \\
\hline Case 1 & 38 & Japanese & Positive & Thyrotoxicosis & 1992 & {$[12]$} \\
\hline
\end{tabular}

"Simultaneous occurrence of postpartum hypopituitarism (Sheehan's syndrome) and transient resolving thyrotoxicosis due to postpartum painless thyroiditis"***

\begin{tabular}{|c|c|c|c|c|c|c|}
\hline Case 2 & 29 & Japanese & Positive & Thyrotoxicosis & 1997 & {$[13]$} \\
\hline \multicolumn{7}{|c|}{ "Painless thyroiditis developed in a patient with Sheehan's syndrome"*** } \\
\hline Case 3 & 30 & French & Positive & Thyrotoxicosis & 2002 & {$[4]$} \\
\hline \multicolumn{7}{|c|}{ "Postpartum autoimmune thyroiditis in a patient presenting with Sheehan's syndrome"*** } \\
\hline Case 4 & 36 & Japanese & Positive & Thyrotoxicosis & & \\
\hline
\end{tabular}

for the development of PPAT. A case of SS with Graves' hyperthyroidism has been also reported [23].

Glucocorticoids are the main endogenous anti-inflammatory agents in vivo, interfering with every step of immune and inflammatory responses [24], and are commonly used in the treatment of autoimmune diseases. The development of autoimmune thyroid diseases has been reported after unilateral adrenalectomy for Cushing's syndrome [10], after bilateral adrenalectomy in a patient with Carney's complex [11] and after removal of ACTH-producing pituitary adenoma in patients with Cushing's disease [11]. The importance of endogenous glucocorticoids in the control of immune conditions is also exemplified by the increased mortality associated with adrenalectomy in rats 
with experimental allergic encephalomyelitis [25]. Transient thyrotoxicosis was also reported to occur after cessation of steroid therapy in a patient with autoimmune thyroiditis and rheumatoid arthritis [12]. Another patient with hypopituitarism, following pituitary apoplexy, developed transient thyrotoxicosis due to painless thyroiditis (autoimmune destructive thyroiditis) [15]. Pituitary apoplexy and SS cause ACTH deficiency and secondary adrenocortical insufficiency (a cortisol decrease). This cortisol decrease may exacerbate autoimmune thyroid diseases. Hypoadrenalism induces immunological alterations, which may be associated with the development of PPAT.

In summary, we experienced a patient with SS who developed PPAT. Postpartum immunological rebounds and hypoadrenalism-induced immunological alterations (or a combination of the two) may have been responsible for the development of PPAT.

\section{Conflict of Interests}

None of the authors have accepted any funding or support from any organization that may gain or lose financially from the results of our study. None of the authors have been employed by any organization that may gain or lose financially from the result of our study.

\section{References}

[1] H. L. Sheehan, "Postpartum necrosis of the pituitary," Journal of Pathology and Bacteriology, vol. 45, no. 2, pp. 189-193, 1937.

[2] Z. Karaca, F. Tanriverdi, K. Unluhizarci, and F. Kelestimur, "Pregnancy and pituitary disorders," European Journal of Endocrinology, vol. 162, no. 3, pp. 453-475, 2010.

[3] J. Ginsberg and P. G. Walfish, "Post partum transient thyrotoxicosis with painless thyroiditis," The Lancet, vol. 1, no. 8022, pp. 1125-1128, 1977.

[4] N. Amino, H. Mori, and Y. Iwatani, "High prevalence of transient post-partum thyrotoxicosis and hypothyroidism," The New England Journal of Medicine, vol. 306, no. 14, pp. 849$852,1982$.

[5] B. Zantour, M. Chadli-Chaieb, A. Maaroufi et al., "Postpartum autoimmune thyroiditis in a patient presenting with Sheehan's syndromeThyrö̈dite auto-immune du post-partum chez une patiente présentant un syndrome de Sheehan," Annales d'Endocrinologie, vol. 63, no. 3, pp. 223-225, 2002.

[6] A. F. Muller, H. A. Drexhage, and A. Berghout, "Postpartum thyroiditis and autoimmune thyroiditis in women of childbearing age: recent insights and consequences for antenatal and postnatal care," Endocrine Reviews, vol. 22, no. 5, pp. 605$630,2001$.

[7] M. W. Groër, "Postpartum thyroiditis," Expert Review of Obstetrics and Gynecology, vol. 3, no. 2, pp. 239-244, 2008.

[8] E. N. Pearce, A. P. Farwell, and L. E. Braverman, "Thyroiditis," The New England Journal of Medicine, vol. 348, no. 26, pp. 2646-2655, 2003.

[9] D. S. Cooper, "Hyperthyroidism," The Lancet, vol. 362, no. 9382, pp. 459-468, 2003.

[10] N. Takasu, I. Komiya, Y. Nagasawa, T. Asawa, and T. Yamada, "Exacerbation of autoimmune thyroid dysfunction after unilateral adrenalectomy in patients with Cushing's syndrome due to an adrenocortical adenoma," The New England Journal of Medicine, vol. 322, no. 24, pp. 1708-1712, 1990.

[11] N. Takasu, N. Ohara, T. Yamada, and I. Komiya, "Development of autoimmune thyroid dysfunction after bilateral adrenalectomy in a patient with Carney's complex and after removal of ACTH-producing pituitary adenoma in a patient with Cushing's disease," Journal of Endocrinological Investigation, vol. 16, no. 9, pp. 697-702, 1993.

[12] H. Maruyama, M. Kato, O. Mizuno, K. Kataoka, and S. Matsuki, "Transient thyrotoxicosis occurred after cessation of steroid therapy in a patient with autoimmune thyroiditis and rheumatoid arthritis," Endocrinologia Japonica, vol. 29, no. 5, pp. 583-588, 1982.

[13] H. Sasaki, H. Shijyo, P. Cugini, T. Kawasaki, and M. Okumura, "Simultaneous occurrence of postpartum hypopituitarism (Sheehan's syndrome) and transient resolving thyrotoxicosis due to postpartum painless thyroiditis," Southern Medical Journal, vol. 85, no. 6, pp. 660-662, 1992.

[14] H. Watanobe and H. Kawabe, "Painless thyroiditis developed in a patient with Sheehan's syndrome," Journal of Endocrinological Investigation, vol. 20, no. 6, pp. 335-337, 1997.

[15] H. Sasaki, O. Ohnishi, T. Okudera, and M. Okumura, "Simultaneous occurrence of transient resolving thyrotoxicosis due to painless thyroiditis, hypopituitarism and diabetes insipidus following pituitary apoplexy," Postgraduate Medical Journal, vol. 67, no. 783, pp. 75-77, 1991.

[16] V. Gasco, G. Corneli, S. Rovere et al., "Diagnosis of adult GH deficiency," Pituitary, vol. 11, no. 2, pp. 121-128, 2008.

[17] H. Atmaca, F. Tanriverdi, C. Gokce, K. Unluhizarci, and F. Kelestimur, "Do we still need the TRH stimulation test?" Thyroid, vol. 17, no. 6, pp. 529-533, 2007.

[18] F. Keleştimur, "Sheehan's syndrome," Pituitary, vol. 6, no. 4, pp. 181-188, 2003.

[19] J. Kaplun, C. Fratila, A. Ferenczi et al., "Sequential pituitary MR imaging in Sheehan syndrome: report of 2 cases," American Journal of Neuroradiology, vol. 29, no. 5, pp. 941943, 2008.

[20] R. Goswami, N. Kochupillai, P. A. Crock, A. Jaleel, and N. Gupta, "Pituitary autoimmunity in patients with Sheehan's syndrome," Journal of Clinical Endocrinology and Metabolism, vol. 87, no. 9, pp. 4137-4141, 2002.

[21] A. De Bellis, F. Kelestimur, A. A. Sinisi et al., "Antihypothalamus and anti-pituitary antibodies may contribute to perpetuate the hypopituitarism in patients with Sheehan's syndrome," European Journal of Endocrinology, vol. 158, no. 2, pp. 147-152, 2008.

[22] M. Komatsu, T. Kondo, K. Yamauchi et al., "Antipituitary antibodies in patients with the primary empty sella syndrome," Journal of Clinical Endocrinology and Metabolism, vol. 67, no. 4, pp. 633-638, 1988.

[23] W. J. Irvine, D. R. Cullen, and K. I. Kirkham, "Sheehan's syndrome associated with thyrotoxicosis and diabetes mellitus," Proceedings of the Royal Society of Medicine, vol. 62, no. 1, p. 40, 1969.

[24] T. R. Cupps and A. S. Fauci, "Corticosteroid-mediated immunoregulation in man," Immunological Reviews, vol. 65, pp. 133-155, 1982.

[25] I. A. M. MacPhee, F. A. Antoni, and D. W. Mason, "Spontaneous recovery of rats from experimental allergic encephalomyelitis is dependent on regulation of the immune system by endogenous adrenal corticosteroids," Journal of Experimental Medicine, vol. 169, no. 2, pp. 431-445, 1989. 


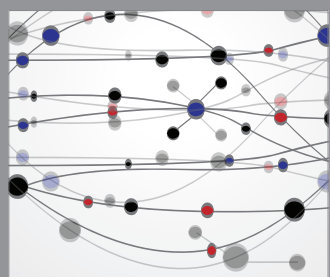

The Scientific World Journal
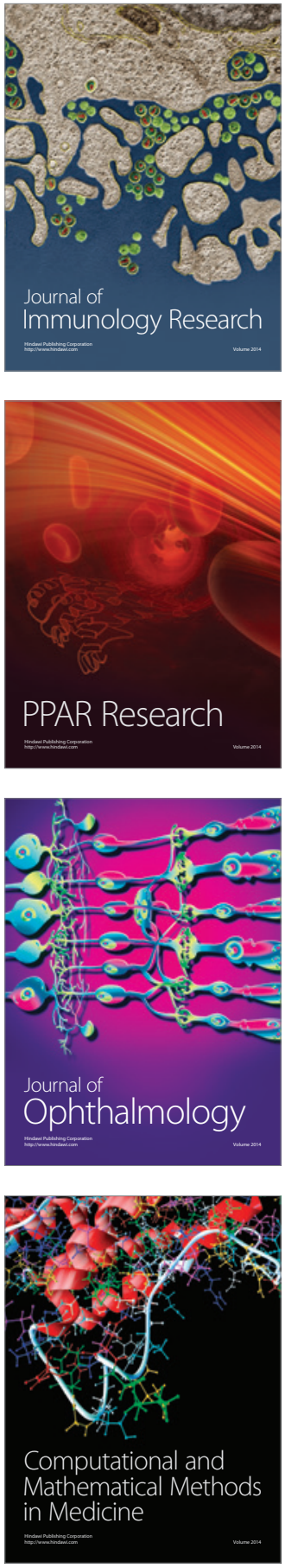

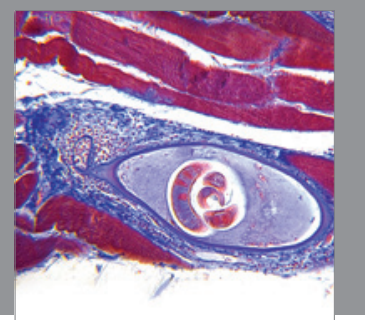

Gastroenterology

Research and Practice
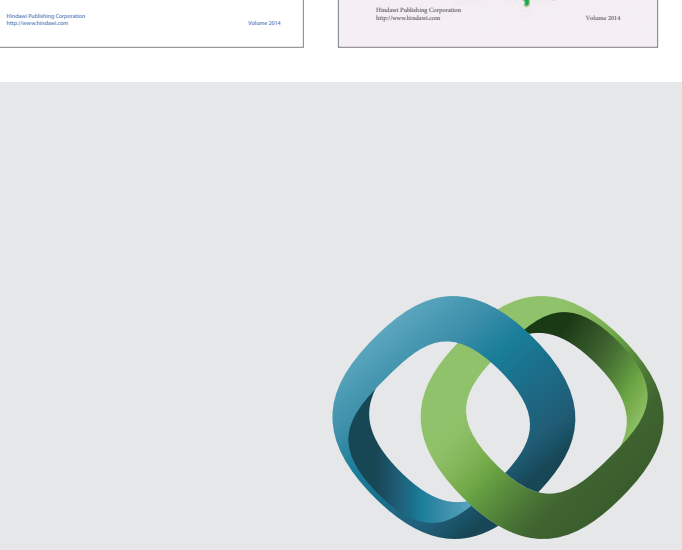

\section{Hindawi}

Submit your manuscripts at

http://www.hindawi.com
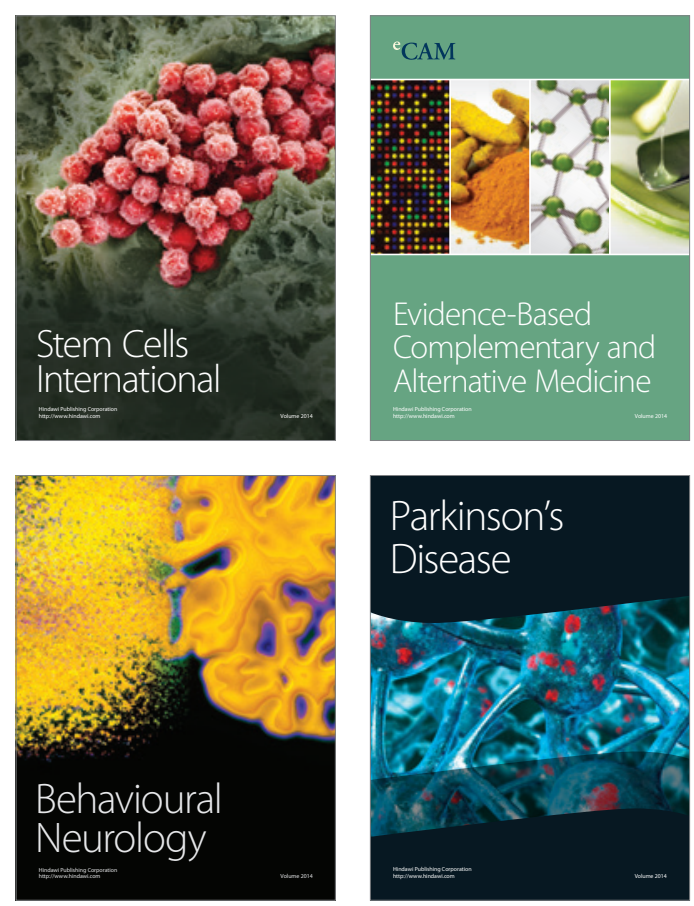

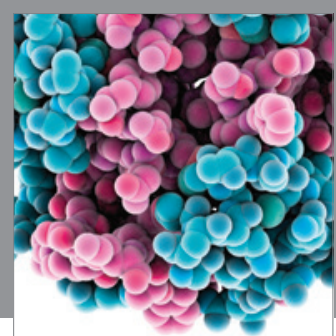

Journal of
Diabetes Research

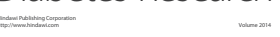

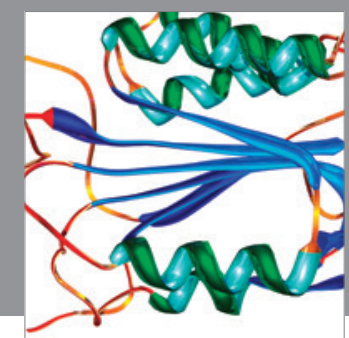

Disease Markers
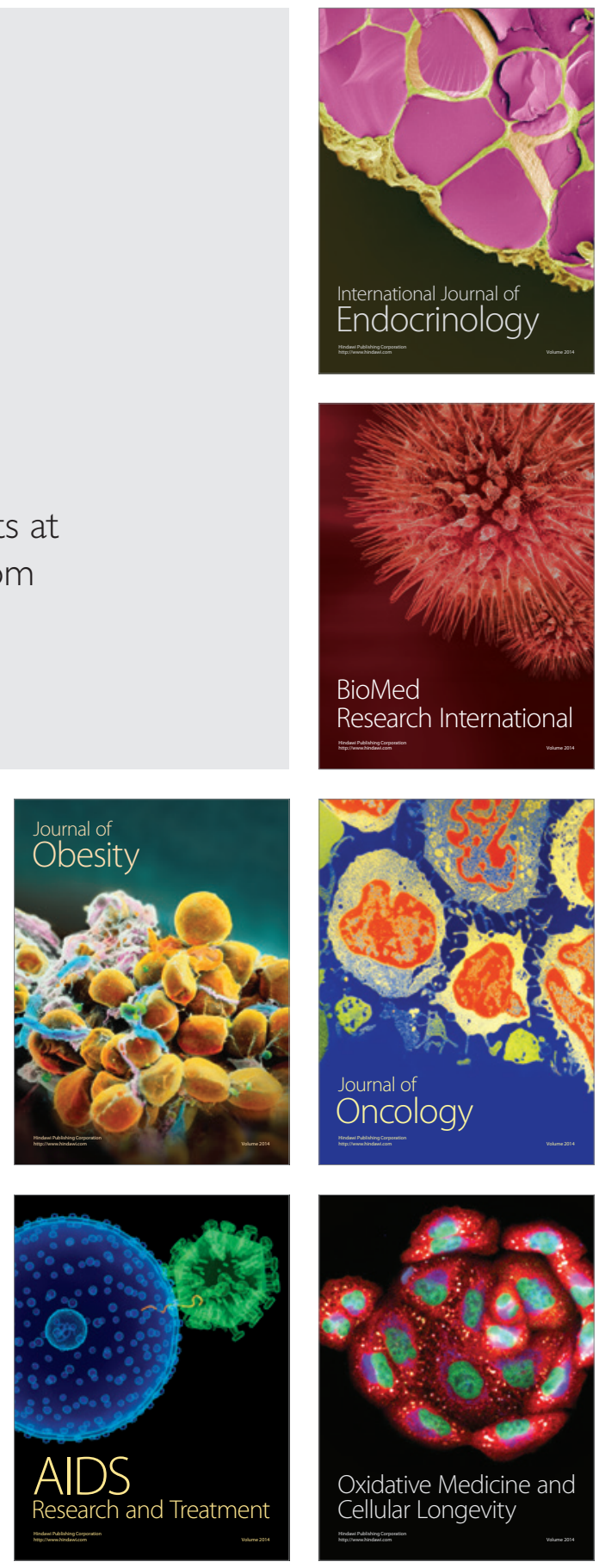